\title{
TOTAL CROSS SECTION FOR OPTICAL SCATTERING BY SPHERICAL CELLS IN SUSPENSION
}

\author{
SHOZO KOGA and TERUYUKI FUJITA \\ The Institute of Applied Microbiology, University of Tokyo \\ Received for publication, March 5, 1960
}

\section{INTRODUCTION}

When light passes through a solution or a suspension, the attenuation of its magnitude is caused by scattering as well as by absorption. In macromolecular chemistry, light scattering has been studied for the purpose of evaluation of molecular weight, while in microbiology it seems to afford a means for the estimation of variations in cell population. Theories have well been developed by RAYLeigh and Gans (1) for the cases in which the diameter of scatterers is smaller than the wave length of light as in macromolecular chemistry. When the diameter of scatterers is larger, as often met in microbial cell suspensions, than the wave length of the rays used, theoretical analysis of scattering phenomena has to be performed in accordance with the Mis's rigorous expression (2). Owing to the lack of an analytically expressed formula, a number of experimental studies have been conducted in the field of microbiology so far without theoretical basis.

In the present paper, it will experimentally be shown that a simplified formula originally given by $\mathrm{HART}_{\mathrm{A} T}$ and MonTRoLl (3) holds well for the total cross section of optical scattering in the suspension of spherical cells with the diameter of $10^{-4} \mathrm{~cm}$ in order. The expressions is much more compact in form and will make as illustrated in the following papers a convenient working basis for the analysis of scattering spectra in cell suspensions.

\section{THEORETICAL}

For the sake of simplicity, unicellular organisms of spherical (or quasispherical) form will be taken. Disregarding, for the present, complicated internal structures, we may assume the cells as optically homogeneous spheres having geometrical radius $a$ and effective refractive index $n_{1}$ in common. This single phase model allows us to apply the same mode of analysis as in non-biological systems. As is well known, numerical computations involved in MIE's scheme is formidably time consuming for the related cases in which the ratio of diameter to wave length amounts to several tens in order. Mathematical tables presented thus far are, moreover, incomplete 
for biological uses due to the fact that relative index of refraction varies in the narrow range close to unity.

An alternative formula was presented by HART and Montroll for the total cross section of soft scatterers as follows:

$$
\sigma_{s} / \pi a^{2}=\left\{\pi x^{2}(m-1)^{2} / 2 m\right\}\left\{\left(m^{4}+6 m^{2}+1\right) \Delta_{1}-2\left(m^{2}+1\right) x^{-2} \Delta_{2}+x^{-4} \Delta_{3}\right\},
$$

where

$$
\Delta_{j}=I_{j}(x[m+1])+I_{j}(x[m-1]),
$$

with

$$
\begin{aligned}
& I_{1}(2 x)=(1 / 2 \pi)-(1 / 8 \pi)\left\{J_{1 / 2}^{2}(2 x)+J_{3 / 2}^{2}(2 x)\right\}, \\
& I_{2}(2 x)=(1 / \pi)\left\{(\sin 4 x / 2 x)-\left(\sin ^{2} 2 x / 4 x^{2}\right)-1+\varphi(4 x)\right\}, \\
& I_{3}(2 x)=(1 / \pi)\left\{2 x^{2}+x \sin 4 x-(5 / 2) \sin ^{2} 2 x+\varphi(4 x)\right\},
\end{aligned}
$$

and

$$
\varphi(z)=\int_{0}^{z}(1-\cos y) d y / y .
$$

On the left hand side of the equation (1), $\sigma_{s}$ is optical cross section for scattering per cell. If multiple scattering does not occur, $\sigma_{s}$ is related to turbidity $(\tau)$ as follows:

$$
\sigma_{s}=\tau / N
$$

where $\tau$ is experimentally given by

$$
\begin{aligned}
I=I_{0} e^{-\tau l} & \\
I_{0}: & \text { Intensity of incident light } \\
I: & \text { Intensity of transmitted light } \\
l: & \text { Length of optical path in } \mathrm{cm} .
\end{aligned}
$$

On the right hand side of the equation (1), relative index of refraction $(m)$ is defined by

$$
\begin{aligned}
m= & n_{1} / n_{0}, \\
& n_{1}: \text { Index of refraction of scatterer } \\
& n_{0}: \text { Index of refraction of suspending medium }
\end{aligned}
$$

and ratio parameter $x$ is defined by

$$
x=2 \pi a / \lambda_{0},
$$

where $\lambda_{0}$ is the wave length of incident light in the suspending medium. Fortunately, relative index of refraction of biological materials is nearly 1 owing to the high content of water in cells. Equation (1) takes, accordingly, a simpler form as follows: 


$$
\begin{gathered}
a_{s}=2-4 \rho^{-1} \sin \rho+4 \rho^{-2}(1-\cos \rho), \\
\rho=2 x(m-1) .
\end{gathered}
$$

Equation (2) is identical with the expression derived independently by vaN DE Hulst (4) for the range $x \gg 1$.

To check the theoretical errors inherent in this approximate formula, the curve of $\sigma_{s}$ computed by equation (2) is compared with the values of $\sigma_{s}$ given in accordance with the MiE's rigorous expression for $m=1.05$ and $m=1.10$ by using PANGonis-Heller-JacoBson's numerical tables (5). The agreement is fairly satisfactory for biological uses as shown in Fig. 1.

The validity of equation (2) may break down for the range $\rho \lesssim 1$. Since, as is well known, RAYLEIGH-GAN's expression represented as follows holds well in this range, equation (2) used in combination with equation (3) will cover the whole range of related problems.

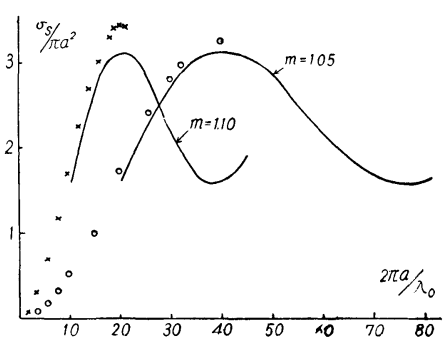

Fig. 1. Approximate curves of $\sigma_{s} / \pi a^{2}$ in accordance with equation (2). Open circles and Crossed bars represent exact values given by the numerical tables (5).

$$
\begin{aligned}
\sigma_{s} / \pi a^{2}= & (m-1)^{2} \phi(x), \\
\phi= & (5 / 2)+2 x^{2}-(\sin 4 x / 4 x)-\left(7 / 16 x^{2}\right)(1-\cos 4 x) \\
& +\left\{\left(1 / 2 x^{2}\right)-2\right\}\left\{0.577+\log 4 x-C_{i}(4 x)\right\}, \\
C_{i}(x)= & -\int_{x}^{\infty}(\cos u / u) d u .
\end{aligned}
$$

For rough estimation of $\sigma_{s}$ in the range $0.2 \lesssim \rho \lesssim 1$, equation (3) may be replaced by the following simpler expression.

$$
\sigma_{s} / \pi a^{2}=(m-1)^{2} 2 x^{2}=(1 / 2) \rho^{2} .
$$

\section{APPARATUS}

In order to obtain experimentally the value of $\sigma_{s}$, the absolute value of turbidity $(\tau)$ must be found for the suspensions, the population of which is so small that multiple scattering has no effect on it. A correct value of $\tau$ can be secured either through the transmission measurement under a narrow beam condition or by the integral measurement of scattered light. An experimental apparatus constructed based on the former principle without the use of lens systems. The collimator part consisted of four identical narrow circular slits as shown in Fig. 2. The size of the slit was determined so as to give a correct value of total scattering with errors less than $5 \%$. If the upper limit of cell size is assumed $10^{-3} \mathrm{~cm}$ in diameter, the al- 


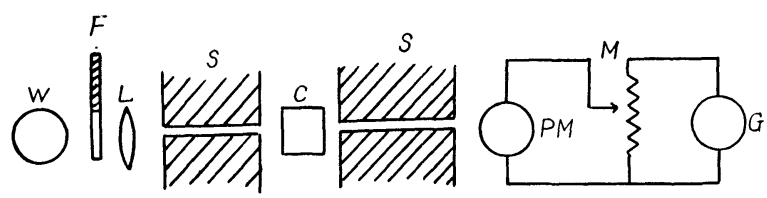

Fig. 2. Experimental apparatus for transmission measurement under narrow beam condition.
$W:$ tungsten lamp
$S: \quad$ slit system $(2 \times 100 \mathrm{~mm})$
$F:$ neutral filter $(\times 1 / 1, \times 1 / 70)$ or interference filter
$C$ : sample cell $(10 \times 10 \times 50 \mathrm{~mm})$
$L$ : collimator lens (as part of light source)
$P M$ : photomultiplier (IP-28)
$G$ : galvanometer $\left(10^{-9} \mathrm{~A} / \mathrm{mm}\right)$
$M$ : galvanometer shunt

lowable maximal diameter of circular slit was estimated to be $2 \mathrm{~mm}$ for the dimensions of apparatus adopted. The larger is the size of a scatterer, the more light is scattered in the forward direction. The estimation was, therefore, roughly made for the case of largest scatterer under consideration by using the Fraunhofer diffraction formula for the angular distribution of scattered light at small angles. By this apparatus the measurement of extinction was possible for suspensions with $\tau$ from 0.1 to 5 in order. This range in $\tau$ covers half a decade in logarithmic population, e.g. $10^{4} \sim 10^{8} \mathrm{cc}$ in the case of Saccharomyces cerevisiae.

\section{EXPERIMENTAL}

(1) Scattering Cross Section

In order to eliminate the effect of multiple scattering, extrapolation to zero population was made by means of the following empirical expression:

$$
\begin{aligned}
\tau_{n}=N /(\boldsymbol{\alpha}+\beta N) & \\
& \tau_{n}: \text { Turbidity at population } N \\
N & : \text { Population density of suspension }
\end{aligned}
$$

or

$$
N / \tau_{n}=\alpha+\beta N
$$

These equations are identical in form with those used for the turbidity of macromolecular solution. As equation (5) takes the form $\tau_{n}=N / \alpha$ when $N$ tends to zero, we get

$$
\sigma_{s}=1 / \alpha .
$$

The value of $\alpha$ will, therefore, be obtained as an intercept on y-axis of $\left(N / \tau_{n}\right)-N$ diagram.

$\tau_{10}\left(\equiv \tau_{n} / 2.30\right)$ observed for suspensions of washed cells was plotted 


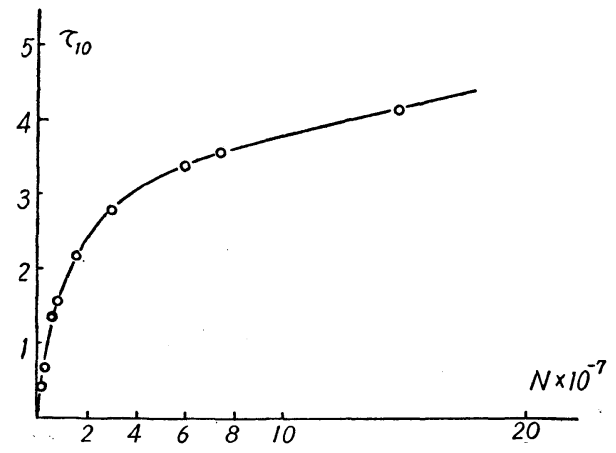

Fig. 3. Optical extinction against population density (Saccharomyces cerevisiae).

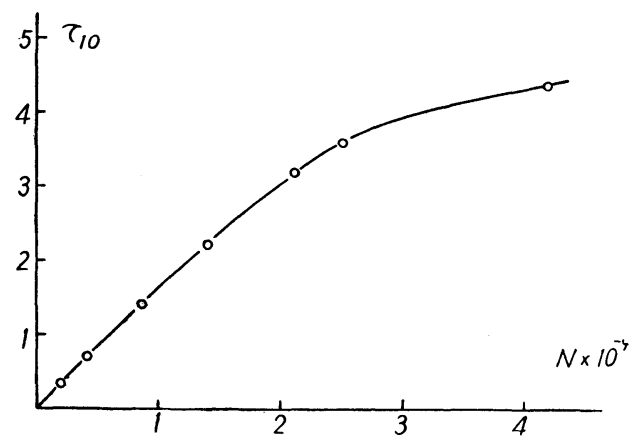

Fig. 5. Optical extinction against population density (Micrococcus luteus).

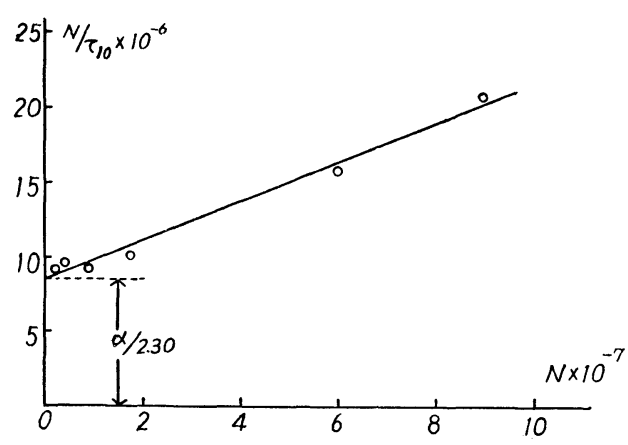

Fig. 7. $N / \tau_{10}$ vs. $N$ (Rhodotorula glutinis).

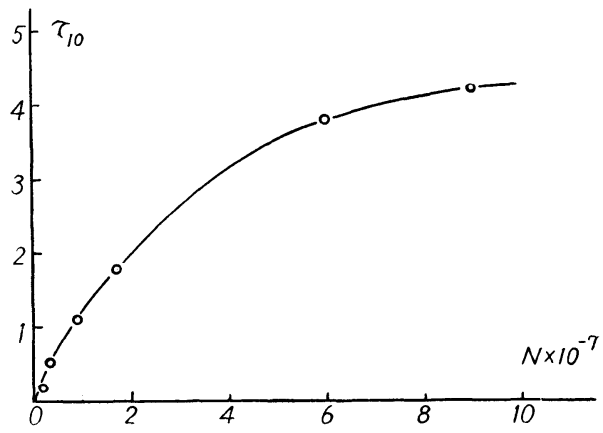

Fig. 4. Optical extinction against population density (Rhodotorula glutinis).

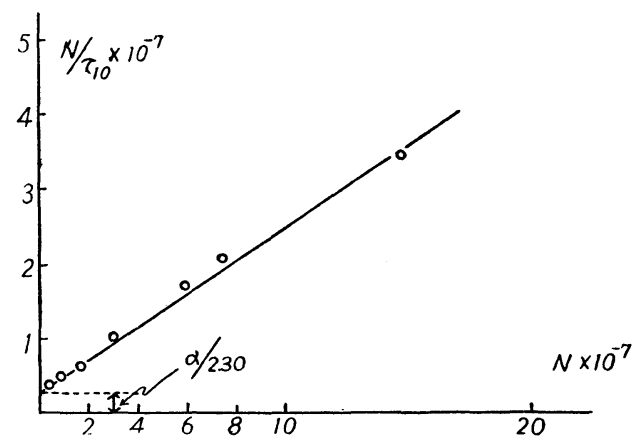

Fig. 6. $N / \tau_{10}$ vs. $N$ (Saccharomyces cerevisiae).

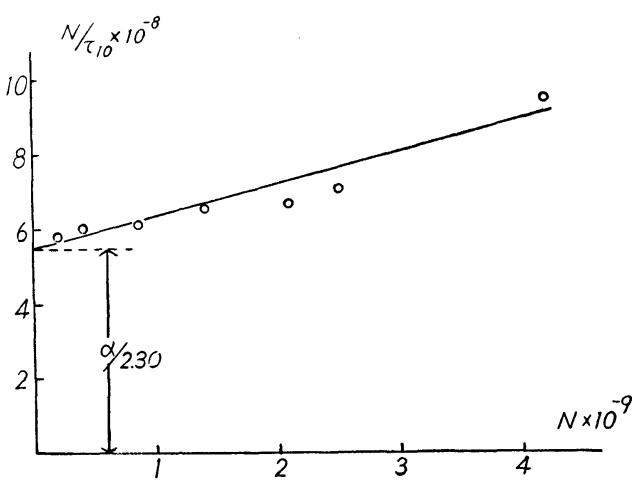

Fig. 8. $N / \tau_{10}$ vs. $N$ (Micrococcus luteus).

against $N$ as shown in Fig. 3, Fig. 4 and Fig. 5. Diagrams of $\left(N / \tau_{10}\right)$ versus $N$ were obtained therefrom as drawn in Fig. 6, Fig. 7 and Fig. 8, giving 
$\sigma_{s}=7.6 \times 10^{-7} \mathrm{~cm}^{2}, 3.3 \times 10^{-7} \mathrm{~cm}^{2}$ and $4.2 \times 10^{-9} \mathrm{~cm}^{2}$ for Saccharomyces, Rhodotorula and Micrococcus, respectively.

\section{(2) Intra-cellular Refractive Index}

Two methods were adopted to obtain the value of cell index of refractions $\left(n_{1}\right)$. Neither of these used any functional form related to the scattering equations described above.

(a) Microscopic Method-Individual cells were observed under a phasemicroscope in the medium of varying index $\left(n_{0}\right)$ of refraction. Phase contrast disappears when $n_{0}$ takes the same value as $n_{1}$. In order to vary the value of $n_{0}$, serum albumin (6) was added to a basal medium. Any sustance with high solubility will do, provided that this molecular weight is sufficiently large to maintain the osmotic balance of the cells. By using a sodium-lamp, 1.40, 1.39 and 1.38 were found as values of $n_{1}$ for Saccharomyces cerevisiae, Rhodotorula glutinis and Micrococcus luteus, respectively.

(b) Minimum Scattering Method-Minimal turbidity points were found for the cell suspensions in the series of media with varying index of refraction $\left(n_{0}\right)$. As is shown in Fig. 9, $n_{1}$ of Saccharomyces cerevisiae was found 1.41 at the wave length of $1 \mu$. Since much larger quantity of adding substances was necessary than in the former case, sucrose was used. The molecular weight of sucrose is not so large as to prevent osmotic volume change in cells, but, since the effect of volume contraction counteracts the effect of increase in refractive index of cells in this case as will be shown in the succeeding paper, the obtained value can be considered correct to a first approximation.

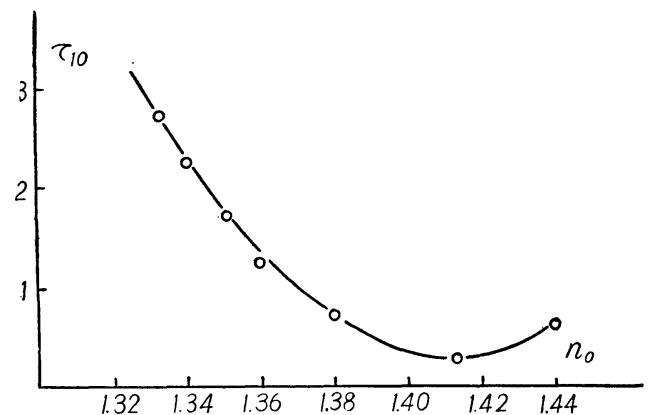

Fig. 9. Optical extinction of cell suspen sions at $1 \mu$ with varying $n_{0}$ (Saccharomyces cerevisiae).

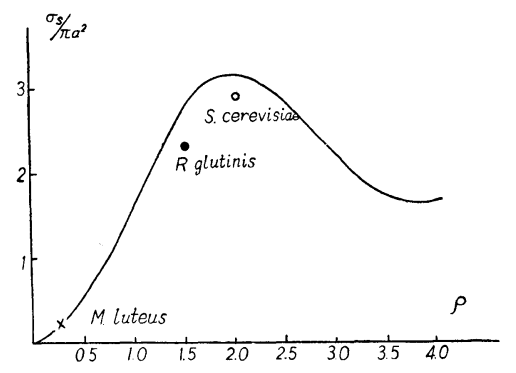

Fig. 10. Observed scattering cross sections compared with the theoretical curve.

(3) Comparison with Theory and Discussion

Taking 1.40 as an average values of refractive index for the cells used, observed values of scattering cross section are well fitted as shown in Fig. 10 by a theoretical curve brawn by means of equation (2) for $\rho>1$ and equation (3) for $\rho<1$. Cell populations and average cell diameters necessary 
for the computation were obtained, thereby, in a similar way as shown in a previous paper $(7)$.

In order to obtain better agreements, many factors, the effects of which were disregarded in the present paper, must be taken into consideration: e.g., departure of cells from spherical in shape, distribution of cell diameters, wave length dispersion of refractive indicies, optical herogeneity of cell content, etc. The approximate representation shown in this paper will, however, prove fairly useful in its simple form for many biological purposes.

\section{ACKNOWLEDGEMENT}

The authors are indebted to Mr. S. Yamaguchi, Mr. K. Komagata and Dr. H. Irzuka for their kindness in supplying strains used in this study.

\section{SUMMARY}

(1) Absolute values of turbidity in cell suspensions were measured under the narrow beam condition, giving, thereby, correct values of scattering cross section per cell.

(2) By using zero method, average indicies of refraction were obtained for Saccharomyces cerevisiae, Rhodotorula glutinis and Micrococcus luteus.

(3) If cells are assumed as homogeneous spheres, the cross section could be computed by RaYleigh-Gans' and Hart-Montroll's expressions. The computed values agreed well with the observed ones for the specimen used.

(4) The equations mentioned above will make, accordingly, a convinient theoretical basis for further studies on scattering of light in cell suspensions as will be shown in succeeding papers.

\section{LITERATURES}

(1) L. Rayleigh: Phil. Mag., 41, 447 (1871); Proc. Roy. Soc., A 90, 219 (1914).

R. GANs: Ann. Phys. Lpz., 76, 39 (1925).

(2) P. MiE: Ann. Phys. Lpz., 25, 3, 771 (1908).

(3) R. W. Hart and E. W. Montroll: J. Appl. Phys., 22, 376, 1278 (1951).

(4) H. C. VAN DE HUlst: Rech. astron. obs. d'Utrecht, 71, part 1 (1946) part 2 (1948).

(5) W. J. Pangonis, W. Heller and A. Jacobson: Tables of Light Scattering Functions for Spherical Particles, Wayne University Press (1957).

(6) R. BARER and F. A. Ross: J. Physiol., 118, 38 (1952).

( 7 ) S. Koga, C. Kuribayashi and K. Nunomura: This Journal 5, 35 (1959). 